\title{
Tangence
}

\section{Élémentaire, mon cher Adso}

\section{Julia Bettinotti et Paul Bleton}

Numéro 38, décembre 1992

Fiction policière et roman actuel

URI : https://id.erudit.org/iderudit/025741ar

DOI : https://doi.org/10.7202/025741ar

Aller au sommaire du numéro

Éditeur(s)

Tangence

ISSN

1189-4563 (imprimé)

1710-0305 (numérique)

Découvrir la revue

Citer cet article

Bettinotti, J. \& Bleton, P. (1992). Élémentaire, mon cher Adso. Tangence, (38),

85-95. https://doi.org/10.7202/025741ar d'utilisation que vous pouvez consulter en ligne.

https://apropos.erudit.org/fr/usagers/politique-dutilisation/ 


\title{
Élémentaire, mon cher Adso *
}

\author{
Julia Bettinotti et Paul Bleton
}

Dans Le nom de la rose d'Umberto Eco, Guillaume de Baskerville, inquisiteur (à la retraite), accompagné par son fidèle Adso, arrive en l'année 1327 dans une très sinistre abbaye où sept moines sont violemment expédiés ad patres l'un après l'autre. L'abbé Abbon prie Baskerville, qui étrenne justement les premières lunettes jamais vues en ce saint lieu, de reprendre du service en découvrant le coupable, et de ramener la paix et l'ordre dans l'abbaye. Le vœeu de l'abbé se réalise au delà de tout espoir, car à la fin du roman il ne reste absolument plus rien, l'abbaye et toutes ses dépendances ayant disparu dans l'ecpyrose finale grâce à l'infâme assassin Jorge de Burgos.

Le nom de la Rose est d'abord une histoire policière mettant en vedette des clones de Sherlock Holmes et de Watson dans les personnages de Baskerville et d'Adso; ce policier semble suivre de très près les vingt règles de Van Dine régissant le roman de détection. Agrémenté par des comptines à la Agatha Christie remplacées ici par les versets de l'Apocalypse, par des cryptogrammes, de l'encre sympatique, du poison, il fait aussi classiquement appel au représentant-borné-de-l'autorité, rôle tenu ici par le grand inquisiteur Bernard Gui, dont les méthodes s'opposent à celles du héros Baskerville-Holmes... En deux mots, le roman d'Eco présente en tout point la trousse à motifs du parfait petit détective de papier.

Ce roman présente aussi du point de vue historique un épisode de la querelle entre le pape d'Avignon et l'empereur, impliquant les ordres bénédictin, franciscain et dominicain. Cela ouvre une autre trousse, celle du Moyen Âge, avec ses signes typiques: abbayes, cathédrales, décors et notamment souterrains gothiques, hérésies, moines sodomites et lubriques, belle "sorcière " qui va être brûlée, etc.

- La recherche dont cette communication est issue a été possible grâce à des subventions du Conseil de recherches en sciences humaines du Canada et du Fonds FCAR. 
86

Mais évidemment les choses ne sont pas si simples: on n'écrit pas un best-seller comme ça, sans problèmes, comme le feraient un quelconque James Michener ou une Judith Krantz, quand on s'appelle Umberto Eco. Élémentaire mon cher Adso? Pas tant que ça, cher Umberto.

Pourtant, l'intention et la position de l'auteur étaient claires dès le début. Dans l'une des rares entrevues qu'il a accordées depuis 1980 au sujet du Nom de la rose (Alfabeta 1983), Eco expliquait:

Je voulais que le lecteur se divertisse. Au moins autant que je me divertissais moi-même. Ce point est capital, et me paraît s'opposer aux conceptions les plus élaborées que nous croyons bâtir sur le roman.

Nul doute que le roman moderne n'ait cherché à dévaloriser le divertissement dû à l'intrigue, pour privilégier d'autres types de divertissement. Grand admirateur de la poétique aristotélicienne, j'ai pour ma part toujours pensé qu'un roman doit divertir malgré tout, même et surtout par son intrigue. ${ }^{1}$

Évidemment, la critique savante ne saurait se laisser berner par le simplisme d'une telle affirmation; aussi a-t-elle plutôt préféré suivre une autre déclaration d'Umberto Eco, devenue du coup prophétique:

Un narrateur n'a pas à fournir d'interprétation à son oeuvre, sinon ce ne serait pas la peine d'écrire des romans, étant donné qu'il sont, par excellence, des machines à générer de l'interprétation. ${ }^{2}$

Ainsi poussée à l'interprétation, la critique, surtout savante, ne s'est pas gênée en réalisant d'ailleurs un autre souhait d'Eco:

Rien ne console plus l'auteur d'un roman que de découvrir les lectures auxquelles il n'avait pas pensé et que les lecteurs lui suggèrent... Je ne dis pas que l'auteur ne puisse découvrir une lecture qui lui semble aberrante, mais dans tous les cas il devrait se taire... ${ }^{3}$

1 Alfabeta, no 49, juin 1983, trad. d'Yves Hersant, cité par Philippe Renard, - Umberto Eco gagne son défi , Critique, nos 447-448 (août 1984), p. 579596.

2 Apostille au Nom de la rose, Le nom de la rose, Paris, Grasset, 1985, p. $509-510$

$3 \quad$ Ibid., p. 511. 
Combinant de façon diabolique la poétique médiévale à celle de l'œuvre ouverte, Eco pousse son lecteur modèle dans les voies, qui sont après tout celles du wbodunit, de la reconnaissance et de l'interprétation. Et d'ailleurs, pourquoi viser un seul lecteur, même modèle, alors qu'on peut en avoir 40 millions? En reliant l'interprétation aux compétences de différentes classes de lecteurs et en leur fournissant, comme dans la pratique médiévale, des histoires qui ne sont pas mutuellement exclusives, mais qui au contraire s'empilent parfaitement les unes sur les autres, Eco réussit le tour de force de construire un texte portant en luimême un modèle scalaire de lecture et de compréhension du texte que différents lecteurs viendront actualiser.

Et c'est ainsi que la critique savante pose sur le texte d'Eco un regard de détective. La critique savante, se plaçant en position d'enquêteur, part à la découverte du vrai texte, du vrai coupable. C'est comme dans un policier: c'est comme si l'auteur avait déguisé son livre, son forfait en suicide, alors que la critique, futée, flaire le meurtre... Les savants collègues d'Umberto Eco se transforment ainsi en Sherlock Holmes ou mieux encore en Nero Wolfe: ne sortant pas de leur tour d'ivoire universitaire, ils cultivent des interprétations aussi exotiques que les orchidées de Nero. La critique savante, à quatre pattes, cherche les "indices " qui vont pointer la "bonne" interprétation du Nom de la rose. Eco, fin diplomate et doté d'un sens de l'humour hors pair, se moque de toute cette agitation de la critique et, laughing all the way to the bank, affirme sans sourciller que tous les sens sont effectivement dans le texte, pourvu que le lecteur veuille bien les y trouver.

Over the past six years, I have repeatedly tried to avoid any statement about the possible interpretations of my novel. My opinion is that a narrator should never provide interpretations for his own text. I do not say this as a radical deconstructionist. On the contrary, I believe that a text... can work as the public parameter of its interpretations. ${ }^{4}$

Il continue:

In several of the essays in this book [Naming the Rose] I found brillant, possible readings of my novel, readings I cannot absolutely challenge because they are rooted in the text and of which I became aware only by reading the readings of my readers.

4 Umberto Eco, "Prelude to a Palimpsest ", in Naming the Rose, Essays on Eco's The Name of the Rose, Jackson, University of Mississippi Press, 1988, p. xi. 
88

Mais la critique savante a probablement exagéré: le dernier texte d'Eco (sa palinodie? la preuve de son agacement?) porte bien le titre Les limites de l'interprétation...

Sans avoir colligé ou analysé de façon exhaustive (travail assez monumental étant donné le succès du roman d'Eco) les critiques parues sur Le nom de la rose, nous nous servons de plusieurs textes, et plus particulièrement de deux recueils, l'un en français, l'autre en anglais, qui comportent, entre autres, des réflexions d'Umberto Eco sur la lecture qu'on a pu faire de ses romans. Il s'agit d'une livraison du Magazine littéraire, parue en 1989, dont le dossier principal était consacré à Umberto Eco (sous le titre $D u$ sémiologue au romancier), et d'un collectif américain portant exclusivement sur Le nom de la rose, soit Naming the Rose: Essays on Eco's The Name of the Rose, paru en 1988 aux Presses de l'Université du Mississippi. À ce corpus critique de base nous avons ajouté quelques articles particulièrement savoureux, dont un assez percutant de Teresa De Lauretis.

D'une part, la critique universitaire est complètement décontenancée par le succès "populaire. du Nom de la Rose, qui devient un best-seller mondial. La critique ne peut se faire à l'idée qu'un grand intellectuel comme Eco ait tout simplement écrit un policier devenu best-seller. Il ne faut pas oublier qu'Eco, en plus de produire des textes du genre $A$ Theory of Semiotics, Sémiotique et philosopbie du langage, a été le théoricien de l'avant-garde italienne des années soixante (Groupe 63) et qu'il a écrit L'ouvre ouverte et Les poétiques de Joyce. De plus, il a fait une thèse sur un sujet sur lequel il n'y a pas à rigoler — Le problème estbétique chez Thomas d'Aquin. Il s'agit là d'états de services absolument impeccables pour un universitaire et dans lesquels tout universitaire peut trouver matière à émulation et stimulation; mais voilà qu'Eco change de cap et que, à l'âge de 50 ans, pris par un démon de midi littéraire, au lieu de nous livrer une Summa Semiotica, il écrit un policier médiéval qui devient un des plus remarquables best-sellers de tous les temps, vendu dans les pharmacies et les super-marchés à côté des Harlequin et de $L a$ cuisine au micro-ondes.

Il arrive qu'un scientifique rencontre un succès public inattendu, même s'il est mérité, avec un de ses essais, écrit Jean-Jacques Brochier, mais qu'un savant spécialisé dans une science humaine aussi difficile, peu connue du public, que la sémiotique, explose dans le monde des lettres avec un roman, le premier, écrit 
lorsqu'il a près de cinquante ans, est une aventure littéraire inédite. Ce savant, c'est Umberto Eco; le roman, Le nom de la rose, deux millions d'exemplaires vendus en Italie, neuf millions dans le reste du monde. 5

Et l'aventure policière commence: le célèbre médiéviste Jacques Le Goff ouvre la voie:

Le Moyen Âge donc, au cœur des deux romans. C'est évident pour le nom de la rose qui, dans une première lecture, littérale, se passe tout entier au début du XIVe siècle. Je dis lecture littérale, car comme les œuvres médiévales, les romans d'Umberto Eco ont plusieurs sens, l'un littéral ou historique, les autres dits, dans le jargon théologique de l'époque, allégorique, moral, anagogique (ou eschatologique, concernant les béatitudes de la vie future) et que nous pouvons appeler - approximativement - spirituels, symboliques - en tout cas cachés. ${ }^{6}$

Le ton est donné: les apparences sont simplettes et trompeuses, la réalité est cryptique. Premier mouvement de la critique donc, le décryptage et l'interprétation symbolique: il n'est pas possible qu'un universitaire de la trempe d'Umberto Eco ait tout simplement écrit un policier, fût-il médiéval. On ira donc chercher dans ce texte toutes sortes de "sens " cachés, de la numérologie à la politique sexuelle d'Eco, dont on ne soupçonnait pas d'ailleurs toutes les ramifications imprévues, comme nous le verrons. La critique se livre donc à une véritable herméneutique du Nom de la rose, traque les différentes significations du texte, tout comme le détective qui s'affaire à découvrir les mobiles du crime.

Le deuxième mouvement, parallèle au premier, est la chasse aux intertextes: il correspond à la découverte, assez facile à faire, il faut le dire, que le texte d'Eco est une rhapsodie de textes, une sorte de patcbwork savant où l'auteur, grâce à son énorme culture, à un système de fiches bien monté et à un sens de l'humour iconoclaste, convoque des éléments culturels qui vont du pape Jean XXIII à l'École de Paris. Et la chasse au whodunit commence: il est évidemment facile de retrouver Voltaire et Peirce dans l'épisode de Brunel, puisque Eco lui-même a écrit un article là-dessus dans The Sign of Three, ou de reconnaître Borges

5 Jean-Jacques Brochier, Magazine littéraire, n 262 (février 1989), présentation du dossier - Umberto Eco , p. 16.

6 Jacques Le Goff, *Forcément médiéval et terriblement moderne *, Magazine littéraire, $\mathrm{n}^{\circ} 262$ (février 1989), p. 30-33. 
dans le bibliothécaire et aveugle Jorge de Burgos, mais il est par contre plus compliqué (mais oh combien valorisant!) de découvrir que, si le nom d'Adso rappelle aux lecteurs naîfs et nombreux celui de Watson, pour les happy few, Eco fait plutôt allusion ici à un Adso moins évident, "Adso's name is certainly calculated by Eco to recall Adso of Montier-en-Der, who wrote an apocalyptic narrative, Libellus de Antechristo, in 954."7 Ach so!

Se dessine ainsi une seconde consécration, tentant d'effacer la consécration du vulgaire: le succès commercial. Seconde consécration qui voudrait rappeler que la Littérature n'est pas affaire de sous mais de Culture, contrairement à ce que cet occasionnel égarement dans la culture de masse aurait pu laisser croire.

La gentrification du succès commercial fonctionne à peu près ainsi : certes, tout le monde a lu Le nom de la rose, mais seuls quelques-uns peuvent avoir accès à l'ensemble des connaissances que le texte convoque et qui permettent des interprétations de plus en plus subtiles. La malheureuse ménagère ou le lector simplex (parangons définitifs du lecteur borné et berné, bien sûr) ne verront dans Le nom de la rose que du feu - celui qui consume tous les livres: un policier classique, beaucoup de cadavres... Et comme chez le divin marquis, entre les meurtres, du verbiage, des textes en latin, des discussions sur l'hérésie qu'il faudra sauter ou lire au plus vite en diagonale. Par contre, les happy few, eux, seront recompensés de leur culture: l'éducation, comme le dit la publicité de certaine université, est une valeur sûre. Les bappy few seront les seuls à savoir qu'à telle page, Eco cite, et pour eux seuls, une phrase de Wittgenstein et, qui plus est, traduite en haut allemand, etc.

Séduite sans doute, la critique doit toutefois rester la critique, assumer qu'elle se doit d'enchaîner quelque forte pensée méta pour dessiller le lecteur moyen - ni tout à fait débile, puisqu'il est bien prêt à voir autre chose qu'un polar dans Le nom de la rose, ni tout à fait aussi éclairé ou subtil que le critique lui-même. Or, cet exercice de finesse, de lecture sagace, de surindividualisation, semble se réduire à des variations sur trois malheureux thèmes critiques, sortes de communautés interprétatives à la

7 Lois Parkinson Zamora, Apocalyptic Visions and Visionaries in The Name of the Rose ", Naming the Rose, op. cit., p. 35. 
Stanley Fish, montrant qu'à un événement dans le socius (la bestsellerisation d'un livre de la culture "choisie "), les réponses possibles dans la sèmiosis particulière des pairs sont très surdéterminées. Les enchaînements possibles sur le nom de la rose ne sont pas infinis; une sorte de matrice des registres de réponse émerge. Chacun des auteurs offre une configuration singulière, aucun ne doit afficher tous les éléments du registre de réponse dans lequel il joue; mais une représentation "en nuage "montrerait bien à la fois l'identité de ces registres et leurs relations.

Commençons par le quatrième de ces trois thèmes critiques, en souvenir du chien dont Sherlock Holmes avait noté qu'il n'avait pas aboyé: l'absence du phénomène étant justement le signe. Le nom de la rose n'est guère rapporté à la classe des polars-en-contexte-religieux ou à celle des polars historiques comme Romanesque, de Ralph McInery ${ }^{8}$. Érudite, la critique savante n'a toutefois pas l'érudition du genre; et si elle ne pratique guère le polar, ce qu'on peut soupçonner, il lui sera d'autant plus facile de voir dans Le nom de la rose autre chose qu'un policier!

Eco est évalué par rapport à lui-même, son œuvre romanesque mesurée à l'aune de ses écrits sémiotiques:

- premier registre:

la stupéfaction du changement de rôle, du sémioticien au best-seller, amène à retourner contre le dernier les outils théoriques que le premier, travaillant sur l'avant-garde et la culture de masse, avait forgé pour l'analyse. La matrice a ici la forme: "Le nom de la rose est une ouvre $\mathrm{X}$ " où $\mathrm{X}$ vaut essentiellement pour "ouverte" ou "fermée".

Communauté interprétative des portiers, des saint Pierre de l'institution littéraire qui se demandent justement et avec acharnement si Eco a bien fait ce qu'il prêchait: il faut qu'une œuvre soit ouverte ou fermée, Le nom de la rose craint-il les courants d'air? Peu importe si L'cuvre ouverte a été publiée il y a trente ans et si son auteur s'est escrimé à répéter pendant trente ans que le concept d'œuvre ouverte est tout simplement une métaphore épistémologique et qu'il n'est pas question de délivrer des certificats d'ouverture ou de fermeture aux ouvres.

8 Traduit dans la Série noire, 1979, sous le titre La cavalcade romaine. 
92

\section{- second registre:}

seuls les gogos s'étonnent de ce que Dr Jekill et Mr Hide soient une même personne, de ce que le professeur émérite soit un aberrant best-seller... La communautés interprétative des bons entendeurs est plus avertie que ça; elle voit dans le vulgaire polar soit la face exotérique d'un enseignement ésotérique, louable effort de vulgarisation, soit l'effet de quelque idée obsédante. La matrice a ici la forme: - la leçon sémiotique du Nom de la rose est.... Ces sémiocomparatistes sont complètement indifférents à l'histoire policière, dont ils ne retiennent que les passages qui permettent de voir que Guillaume de Baskerville fait de l'abduction comme C. S. Peirce, ils vont chercher des analogies *bord à bord "entre les idées sur les codes et les signes exprimées dans le roman et celles traitées par Eco dans Theory of Semiotics et Sémiotique et philosopbie du langage. Au diable les tautologies: il est évident que les théories sont les mêmes, étant donné qu'elles appartiennent au même auteur, qui ne semble pas avoir pour le moment des troubles de la personnalité et qui le dit d'ailleurs: *[...] on a trouvé par la suite dans mon premier roman, comment dire...? une allégorie de mes principes sémiotiques" 9 .

Et il ajoute, dans la préface à Naming the Rose,

I never suspected that my novel was so consistent with my research in semiotics, because I told my story by accepting a split personality, and I did not (consciously) try to put in my novel the theories I had developed in my scholarly writings. I will admit, though, that even the most schizophrenic personality cannot be as split as that. A good reader can understand the relationships between my various books better than I can. ${ }^{10}$

\section{- troisième registre:}

contre cette démesure de la mesure par rapport à l'auteur, une autre conception remet les pendules à l'heure. Le nom de la rose, devenu best-seller, jeté sur le marché du discours social, transforme Eco en personnage public, en nom propre idéologiquement significatif, en désignateur rigide de positions idéologiques... Il est cette fois évalué par rapport à des discours constitués déjà là; le

9 Umberto Eco, "De L'ceuvre ouverte au Pendule de Foucault", propos recueillis par Jean-Jacques Brochier et Mario Fusco, Magazine littéraire, $n^{\circ} 262$ (février 1989), p. 20.

10 Umberto Eco, *Prelude to a Palimpsest *, Naming the Rose, op. cit., p. XII. 
projet global, la pseudo-unité d'une carrière et d'une identité individuelle sont moins déterminants que le choix du camp idéologique trahi par tels ou tels énoncés du best-seller... Communauté interprétative des idéologues, des lecteurs de symptômes, de loin la catégorie la plus fréquentée, elle regroupe tous ceux et celles qui voient dans cette histoire médiévale une allégorie des temps modernes et qui attribuent à Eco des "vouloir-dire "variés. La matrice a ici la forme: "Eco est un $W$ " où $W$ vaut pour... non, je sais, vous ne nous croirez pas; nous sommes en effet là dans la catégorie la plus loufoque avec les interprétations les plus farfelues: grâce aux représentants de cette veine on peut apprendre qu'Eco préfigure, nouveau Jean de Patmos, la fin de ce monde corrompu; que les frères franciscains et les fraticelli du Moyen Âge sont bien les Brigades Rouges d'aujourd'hui; qu'Eco est - au choix - un fasciste anti-gay, un macho et un closet bomosexual, pour ne pas dire qu'il est absolument et résolument féministe (refresbingly feminist), etc.!

Nous allons vous donner quelques exemples de ces trois voies empruntées par la critique.

Les Saint-Pierristes: de la fermeture à l'ouverture en passant par l'entrebaillé. Il s'agit d'un roman, dit Maria Corti «qui est si lucidement construit et tellement clos qu'il respecte, d'une façon tout à fait exceptionnelle aujourd'hui même les unités aristotéliciennes de temps, de lieu, d'action "11.

Alors que Jocelyn Mann est péremptoire: “This is, after all, an open novel..."12

Pour les sémiocomparatistes il n'y a aucun problème d'identification: ainsi l'usage que fait Eco des anagrammes dans $L e$ nom de la rose est évidemment un hommage à Saussure, comme la thématisation du rire un hommage à Bakhtine et l'incendie de la bibliothèque une évidente allusion à Derrida. Plus subtil encore, Robert Caserio propose cette clé d'interprétance: Jorge est assimilé au "malignant referent * et en fait la lutte entre William (il s'agit d'Eco, bien entendu) et Jorge (qui devient "the referential

11 Maria Corti, *E un' opera chiusa*, L'espresso, 10 octobre 1980. Nous traduisons.

12 Jocelyn Mann, *Traversing the Labyrinth: The Structures of Discovery in Eco's The Name of the Rose", Naming the Rose, op. cit., p. 141. 
fallacy ") représente la lutte d'Eco contre le référent telle qu'elle s'exprime dans Theory of Semiotics ${ }^{13}$.

Pour ce qui est des idéologues de la troisième catégorie, un exemple suffit, celui de Philippe Renard:

Dans la foire aux antiquaires apprêtée par Umberto Eco, nous retrouvons toutes les problématiques de notre temps: le marxisme avec l'aspiration à la rédemption spirituelle mais surtout matérielle de l'homme, avec la figure du sous-prolétaire du XIV' siècle Salvatore - qui ne domine pas le langage, comme nos immigrés; le fantasme de 68 avec le citoyen révolutionnaire - Remigio qui aspire à la fête de l'égalité; mais surtout les Brigades Rouges que sont déjà les troupes de Fra Dolcino. ${ }^{14}$

Que reste-t-il de ce merveilleux policier médiéval d'Eco, une fois que la critique savante est passée par là comme le proverbial rouleau? Nous nous permettons ici une réponse quelque peu allégorique, comme il se doit, trouvée d'ailleurs dans une anecdote rapportée par Eco lui-même: à vous de trouver le coupable.

Alors qu'on voulait savoir quelle était l'intrigue du nouveau roman d'Umberto Eco et que l'on avait inventé quatre ou cinq possibilités d'histoires différentes, Eco lui-même, à peine narcissique, s'amusait de ce qu'on disait de son roman à venir; il écrivit un texte là-dessus dans l'hebdomadaire L'espresso. Il y est question, entre autres, de l'intrigue suivante:

\section{L'étrange aventure de $M$. Baskerville}

Baskerville, un éditeur de grand caractère, a eu, pour éviter de payer des droits d'auteur, une idée extraordinaire. Il prend un livre en anglais, le fait traduire en italien, donne la version italienne à un traducteur bulgare, la version bulgare à un traducteur chinois, la version chinoise à un traducteur italien et obtient ainsi un roman absolument inédit. Le drame se produit quand, ayant eu affaire à un texte du courant de la "pensée faible", et après l'avoir fait passer à travers huit traductions successives, il se retrouve toujours avec une pensée aussi faible. Il avale de l'encre d'imprimerie et meurt dans d'atroces souffrances. ${ }^{15}$

13 Robert Caserio, *Why the Novel Matters. II. The Name of the Horse: Hard Times, Semiotics, and the Supernatural *, Novel, vol. XX, $\mathrm{n}^{\circ} 1,1982$, p. 5-23.

14 Philippe Renard, * Umberto Eco gagne son défi , op. cit., p. 582.

15 Cité dans Umberto Eco, "Mon nouveau Roman", Magazine littéraire, $n^{\circ} 262$, p. 29. 
Et pourquoi pas d'ailleurs? Eco le dit lui-même: "L'auteur devrait mourir après avoir écrit. Pour ne pas gêner le cheminement du texte. ${ }^{16} \mathrm{La}$ critique le prend sans doute au mot, puisque, comme on le voit, elle s'évertue à le faire mourir... de rire.

16 Umberto Eco, Apostille au Nom de la rose, op. cit., p. 512. 